\title{
Erratum to: Frailty has a stronger association with inflammation than age in older veterans
}

\author{
P. Van Epps ${ }^{1,2^{*}}$, D. Oswald ${ }^{2}$, P. A. Higgins ${ }^{1,4}$, T. R. Hornick ${ }^{1,3}$, H. Aung ${ }^{1,2}$, R. E. Banks ${ }^{1}$, B. M. Wilson ${ }^{1}$, C. Burant ${ }^{1,4}$, \\ S. Gravenstein ${ }^{3}$ and D. H. Canaday ${ }^{1,2}$
}

\section{Erratum}

In the original publication of this article [1], the author S. Graventstein was listed incorrectly. The correct spelling should have been S. Gravenstein.

\begin{abstract}
Author details
'Geriatric Research, Education, and Clinical Center, Louis Stokes Cleveland VA Medical Center, Cleveland, OH, USA. ${ }^{2}$ Division of Infectious Disease, Case Western Reserve University, Cleveland, OH, USA. ${ }^{3}$ Division of Geriatrics, Department of Medicine, Case Western Reserve University, Cleveland, $\mathrm{OH}$, USA. ${ }^{4}$ School of Nursing, Case Western Reserve University, Cleveland, $\mathrm{OH}$, USA.
\end{abstract}

Received: 24 January 2017 Accepted: 13 February 2017

Published online: 16 February 2017

\section{References}

1. Van Epps P, et al. Frailty has a stronger association with inflammation than age in older veterans. Immun Ageing. 2016;13:27. doi:10.1186/s12979-016-0082-z.

\footnotetext{
*Correspondence: puja.vanepps@va.gov

'Geriatric Research, Education, and Clinical Center, Louis Stokes Cleveland VA Medical Center, Cleveland, OH, USA

${ }^{2}$ Division of Infectious Disease, Case Western Reserve University, Cleveland, $\mathrm{OH}$, USA
} 